Article

\title{
Internet Survey of Awareness and Behavior Related to HPV Vaccination in Japan
}

\author{
Risa Kudo ${ }^{1}$, Masayuki Sekine ${ }^{1, *} \mathbb{( D}$, Manako Yamaguchi ${ }^{1}$, Megumi Hara ${ }^{2}$, Sharon J. B. Hanley ${ }^{3}$, Yutaka Ueda ${ }^{4}(\mathbb{D}$, \\ Asami Yagi ${ }^{4}$, Sosuke Adachi ${ }^{1}$, Megumi Kurosawa ${ }^{1}$, Etsuko Miyagi ${ }^{5}$ and Takayuki Enomoto ${ }^{1}$ \\ 1 Department of Obstetrics and Gynecology, Niigata University Graduate School of Medical and Dental Sciences, \\ Niigata 951-8510, Japan; pearpear@med.niigata-u.ac.jp (R.K.); manako0131@med.niigata-u.ac.jp (M.Y.); \\ sadachi@med.niigata-u.ac.jp (S.A.); m-kurosawa@med.niigata-u.ac.jp (M.K.); enomoto@med.niigata-u.ac.jp (T.E.) \\ 2 Department of Preventive Medicine, Faculty of Medicine, Saga University, Saga 849-8501, Japan; \\ harameg@cc.saga-u.ac.jp \\ 3 Department of Obstetrics and Gynecology, Hokkaido University Graduate School of Medicine, \\ Sapporo 060-8638, Japan; sjbh1810@med.hokudai.ac.jp \\ 4 Department of Obstetrics and Gynecology, Osaka University Graduate School of Medicine, Suita 565-0871, Japan; \\ y.ueda@gyne.med.osaka-u.ac.jp (Y.U.); a.yagi@gyne.med.osaka-u.ac.jp (A.Y.) \\ 5 Department of Obstetrics and Gynecology, Yokohama City University School of Medicine, \\ Yokohama 236-0004, Japan; emiyagi@yokohama-cu.ac.jp \\ * Correspondence: masa@med.niigata-u.ac.jp; Tel.: +81-25-227-2320; Fax: +81-25-227-0789
}

\section{check for}

updates

Citation: Kudo, R.; Sekine, M.; Yamaguchi, M.; Hara, M.; Hanley, S.J.B.; Ueda, Y.; Yagi, A.; Adachi, S.; Kurosawa, M.; Miyagi, E.; et al. Internet Survey of Awareness and Behavior Related to HPV Vaccination in Japan. Vaccines 2021, 9, 87. https://doi.org/10.3390/vaccines 9020087

Academic Editor: Giuseppe La Torre Received: 21 December 2020

Accepted: 21 January 2021

Published: 25 January 2021

Publisher's Note: MDPI stays neutral with regard to jurisdictional clai$\mathrm{ms}$ in published maps and institutional affiliations.

Copyright: (C) 2021 by the authors. Licensee MDPI, Basel, Switzerland. This article is an open access article distributed under the terms and conditions of the Creative Commons Attribution (CC BY) license (https:// creativecommons.org/licenses/by/ $4.0 /)$.

\begin{abstract}
Recommendations for HPV vaccines were suspended in 2013 due to unfounded safety fears in Japan. We aimed to clarify the differences between vaccinated and unvaccinated females in their awareness, knowledge, and behaviors toward cervical cancer, HPV vaccination and sex. Questionnaires were administered online to women aged 16 to 20 . We conducted investigations for the following: awareness, knowledge, and actions for cervical cancer, HPV vaccination, and sexual activity, as well as items related to participants' social background. The survey in 828 girls revealed three points. The first is that more than half of the surveyed Japanese girls had poor knowledge about cervical cancer screening, HPV, or HPV vaccines. The second is that those in the unvaccinated group had a particularly poor knowledge of the subject and tended to have higher sexual activity. The final is that only $0.5 \%$ of the girls experienced changes in awareness about sexual activity after vaccination. In conclusion, this is the first large-scale survey analyzing the association between HPV vaccination and sexual activity in Japanese girls. Not only do unvaccinated girls not benefit from vaccines, but they also tend to engage in high-risk sexual behavior, and thus it is even more important to provide information on the effectiveness of vaccines and the usefulness of cancer screening.
\end{abstract}

Keywords: HPV vaccination; cervical cancer; Internet survey; sexual behavior; cancer screening

\section{Introduction}

The bivalent vaccine and quadrivalent vaccine were approved as human papillomavirus (HPV) vaccines for primary prevention against cervical cancer in Japan in 2009 and 2011, respectively [1]. Public funding for HPV vaccinations for women aged 13-16 years was initiated in 2010 by municipalities and in April 2013 it was included in the national immunization program (NIP) for women aged 12-16 years. HPV vaccination rates in Japan are high $(70 \%)$ despite the vaccine not being administered within school-based programs, but individually in clinics [2,3]. However, around the same time, there was sensationalized reporting on the various symptoms which were misunderstood to occur following immunization. This prompted the Ministry of Health, Labor, and Welfare to suspend proactive recommendations for the HPV vaccine in June 2013 [1]. Vaccination rates dropped sharply to $<1 \%$ in less than a year [2]. To this day, the vaccination rate continues to be close to zero despite the HPV vaccine still being included in the NIP and women within the target age range being able to receive $H P V$ vaccination for free $[3,4]$. 
HPV vaccination is recommended as a national project in many countries around the world. Regarding the effectiveness of HPV vaccines, many papers reported showing the preventive effect on the HPV infection and precancerous lesions [1,5-8], and, in addition, a paper showing the effect of reducing invasive cervical cancer was published by Sweden in October 2020 for the first time in the world. HPV vaccination has been shown to reduce the risk of invasive cervical cancer by $63 \%$, and vaccination under the age of 17 years reduces the risk by $88 \%$ [9]. A Cochrane review that analyzed safety of the HPV vaccine reported that HPV vaccination increased swelling at the site of injection, but did not increase serious adverse reactions [10]. The WHO continuously evaluates the latest data on HPV vaccines worldwide and reports that no safety issues have been found that would require changes to HPV vaccine recommendations [11]. They conclude that all bivalent, tetravalent, and 9valent vaccines show excellent safety and efficacy data, and each country has published a position paper on HPV vaccination and a guideline on cervical cancer screening [12,13].

Cervical cancer is on the rise in Japan in terms of both morbidity and mortality. The increasing morbidity rate, particularly among young women of reproductive age, has been considered problematic alongside late births [14]. Rates of cervical cancer screening as secondary prevention in Western countries are 60-80\%, whereas those in Japan are 30-40\%, with rates among those in their early $20 \mathrm{~s}$ in particular at $10 \%$ or less [15]. As the only country with increasing rates of cervical cancer, Japan is behind the rest of the world.

It is anticipated that seven years after suspension of proactive recommendations, simply reinstating proactive recommendations at the national level will not sufficiently recover vaccination rates that have rapidly fallen to almost zero. Other than the concern for adverse events, people opposed to resuming HPV vaccination recommendations argue that girls vaccinated against HPV would become more sexually active and less willing to undergo cervical cancer screening, eventually leading to no desirable reduction in cervical cancer morbidity risks [16].

This study aimed to clarify the differences between vaccinated and unvaccinated females in their awareness, knowledge, and behaviors toward cervical cancer, cervical cancer screening, HPV vaccination, and sex and investigated methods for recovering the drastically reduced vaccination rate by analyzing changes after HPV vaccination.

\section{Materials and Methods}

\subsection{Internet Survey}

Questionnaires were administered online to women aged 16 to 20 across Japan (i.e., those who belonged to a generation where publicly funded HPV inoculations have been conducted) from 5 February 2015 to 12 February 2015. Questionnaires were emailed to target-age women who were registered as monitoring members on Macromill. Basic attributes-such as sex, age, occupation, place of residence, and marital and child status-were registered for monitoring members on Macromill. In this study, we used vaccination and willingness to answer questionnaires as allocation factors and selected enrollees so that the age composition of the vaccinated and unvaccinated groups would be equal. We conducted investigations for the following in both vaccinated and unvaccinated groups: awareness, knowledge, and actions for cervical cancer, cervical cancer screening, HPV vaccination, and sexual activity, as well as items related to participants' social background such as socioeconomic status, drinking history, and smoking history.

\subsection{Statistical Analysis}

The data were analyzed using SPSS (Ver25) (IBM, Chicago, IL, USA). Categorical data are presented as absolute numbers and percentages. Continuous data are presented as the means \pm standard deviation. Pearson's chi-squared test, Fisher's exact test, Student's t-test, the Mann-Whitney test, logistic regression analysis were used to check for differences in baseline characteristics between the vaccinated and unvaccinated populations. Univariate and multivariate logistic regression analyses were conducted to assess awareness and knowledge for HPV vaccine, cervical cancer, and cervical cancer screening. Statistical anal- 
ysis of knowledge was evaluated using logistic regression analysis. The three answers, "savvy", "vague", and "none", were evaluated as 2, 1, and 0 points, respectively, and scaled. The odds ratio in the vaccinated group was calculated by univariate analysis with the unvaccinated group as the reference level. After that, multivariate analysis was performed on the items for which a significant difference was found in the univariate analysis and the items with strong correlation were evaluated. A $p$-value of $<0.05$ was considered statistically significant. The questionnaire study was conducted anonymously. The subjects were fully informed of the aim of this study. The answers who gave consent filled out the questionnaire. The study was approved by the Ethics Review Board of Niigata University Graduate School of Medical and Dental Sciences (Approval Number 2015-1866).

\section{Results}

\subsection{Background of Participants}

We received questionnaire responses from 828 people. There were 414 people each for the vaccinated and unvaccinated groups. The age distribution was as follows: 16 years, 86 people (10.4\%); 17 years, 132 (15.9\%); 18 years, $228(27.5 \%) ; 19$ years, $274(33.1 \%)$; and 20 years, $108(13.0 \%)$ (Table 1$)$. The survey was conducted so as not to be biased for different parts of Japan, and there were no significant differences regarding the place of residence in the vaccinated and unvaccinated groups. Marriage was significantly more common in the unvaccinated group compared to the vaccinated group, with $32(7.7 \%)$ versus 8 people $(1.9 \%)$, respectively $(p<0.001)$. There were no significant differences in sexual experience between the vaccinated and unvaccinated groups, with 135 (32.6\%) versus $148(35.7 \%)$ people, respectively $(p=0.39)$. The percentage of students was significantly higher in the vaccinated group compared to the unvaccinated group, with $390(94.2 \%)$ people versus 359 $(86.7 \%)$, respectively $(p<0.001)$. As for life history, smoking history was significantly more frequent in the unvaccinated group $(p=0.014)$. Among the participants who were registered as students, over $60 \%$ responded with "unknown" for their annual family income, and no significant differences were found between the vaccinated and unvaccinated groups $(p=0.108)$. To gauge economic status, participants were asked whether they had attended cram schools; attendance was significantly higher in the vaccinated group compared to the unvaccinated group, with 276 people $(66.7 \%)$ versus $238(57.5 \%)$, respectively $(p=0.001)$.

Table 1. Background of participants in this Internet survey.

\begin{tabular}{|c|c|c|c|c|c|}
\hline \multirow{2}{*}{ Age (years) } & \multicolumn{2}{|c|}{ Vaccinated $(n=414)$} & \multicolumn{2}{|c|}{ Unvaccinated $(n=414)$} & \multirow[t]{2}{*}{$p$-Value } \\
\hline & & & & & \\
\hline 16 & 43 & $(10.40 \%)$ & 43 & $(10.40 \%)$ & $1.00 *$ \\
\hline 17 & 66 & $(15.90 \%)$ & 66 & $(15.90 \%)$ & \\
\hline 18 & 114 & $(27.50 \%)$ & 114 & $(27.50 \%)$ & \\
\hline 19 & 137 & $(33.10 \%)$ & 137 & $(33.10 \%)$ & \\
\hline 20 & 54 & $(13.00 \%)$ & 54 & $(13.00 \%)$ & \\
\hline \multicolumn{6}{|l|}{ Marital status } \\
\hline married & 8 & $(1.90 \%)$ & 32 & $(7.70 \%)$ & $<0.001$ * \\
\hline unmarried & 406 & $(98.10 \%)$ & 382 & $(92.30 \%)$ & \\
\hline \multicolumn{6}{|l|}{ Sexual experience } \\
\hline yes & 135 & $(32.60 \%)$ & 148 & $(35.70 \%)$ & $0.39 *$ \\
\hline no & 255 & $(61.60 \%)$ & 246 & $(59.40 \%)$ & \\
\hline unanswered & 24 & $(5.80 \%)$ & 20 & $(4.80 \%)$ & \\
\hline \multicolumn{6}{|l|}{ Occupation } \\
\hline student & 390 & $(94.20 \%)$ & 359 & $(86.70 \%)$ & $<0.001 *$ \\
\hline employed & 16 & $(3.90 \%)$ & 25 & $(6.00 \%)$ & \\
\hline housewife & 8 & $(1.90 \%)$ & 30 & $(7.20 \%)$ & \\
\hline \multicolumn{6}{|l|}{ Smorking history } \\
\hline yes & 30 & $(7.20 \%)$ & 51 & $(12.30 \%)$ & 0.014 * \\
\hline no & 384 & $(92.80 \%)$ & 363 & $(87.70 \%)$ & \\
\hline
\end{tabular}


Table 1. Cont.

\begin{tabular}{|c|c|c|c|c|c|}
\hline & \multicolumn{2}{|c|}{ Vaccinated $(n=414)$} & \multicolumn{2}{|c|}{ Unvaccinated $(n=414)$} & \multirow[t]{2}{*}{$p$-Value } \\
\hline 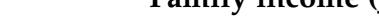 & & & & & \\
\hline$<2$ milion & 41 & $(9.90 \%)$ & 44 & $(10.60 \%)$ & $0.108^{+}$ \\
\hline 2-4 milion & 30 & $(7.20 \%)$ & 45 & $(10.90 \%)$ & \\
\hline 4-6 milion & 22 & $(5.30 \%)$ & 22 & $(5.30 \%)$ & \\
\hline 6-10 milion & 32 & $(7.70 \%)$ & 30 & $(7.20 \%)$ & \\
\hline 10 milion< & 12 & $(2.90 \%)$ & 4 & $(1.00 \%)$ & \\
\hline unknown & 277 & $(66.90 \%)$ & 269 & $(65.00 \%)$ & \\
\hline \multicolumn{6}{|c|}{ Economic circumstances (self-judgment) } \\
\hline very good & 70 & $(16.90 \%)$ & 57 & $(13.80 \%)$ & $0.039 \ddagger$ \\
\hline good & 112 & $(27.10 \%)$ & 107 & $(25.80 \%)$ & \\
\hline normal & 158 & $(38.20 \%)$ & 160 & $(38.60 \%)$ & \\
\hline poor & 64 & $(15.50 \%)$ & 65 & $(15.70 \%)$ & \\
\hline very poor & 10 & $(2.40 \%)$ & 25 & $(6.00 \%)$ & \\
\hline \multicolumn{6}{|c|}{ Attending cram school } \\
\hline yes & 276 & $(66.70 \%)$ & 238 & $(57.50 \%)$ & $0.001 *$ \\
\hline no & 138 & $(33.30 \%)$ & 168 & $(40.60 \%)$ & \\
\hline unknown & 0 & 0 & 8 & $(1.90 \%)$ & \\
\hline
\end{tabular}

${ }^{*}$ Chi-squared test; ${ }^{\dagger} t$-test (exclude "unknown"); ${ }^{\ddagger} t$-test.

\subsection{Knowledge of Cervical Cancer Screening and HPV Vaccination}

Two items for cervical cancer screening, two for cervical cancer, two for HPV, and one for the effects of HPV vaccination are shown (Table 2, Supplemental Table S1). Of these, the vaccinated group had significantly more knowledge of the following items: "Necessity of regular cancer screening" (odds ratio $(\mathrm{OR})=1.29,95 \%$ confidence interval $(\mathrm{CI}): 1.08$ to $1.55, p=0.005)$, "Cervical cancer negatively affects pregnancy" (OR $=1.33,95 \%$ CI: 1.11 to $1.60, p=0.002$ ), "The virus leading to cervical cancer infects during sexual intercourse" $(\mathrm{OR}=1.19,95 \%$ CI: 1.00 to $1.42, p=0.048)$, and "HPV vaccination is effective for cervical cancer prevention" (OR $=2.03,95 \% \mathrm{CI}: 1.11$ to $1.60, p<0.001)$. The most relevant knowledge of HPV vaccination was the effectiveness of the HPV vaccine. Only two items relating to cervical cancer were known by over $50 \%$ of participants, those items were "Cervical cancer negatively affects pregnancy" and "Cervical cancer may cause death." It was noteworthy that only $8.9 \%$ of the unvaccinated knew that "HPV vaccination is effective for cervical cancer prevention."

Table 2. Knowledge of cervical cancer screening and HPV vaccination.

\begin{tabular}{|c|c|c|c|c|c|c|}
\hline \multirow{2}{*}{ Question } & \multicolumn{3}{|c|}{ Univariate Analysis } & \multicolumn{3}{|c|}{ Multivariate Analysis } \\
\hline & OR * & $95 \% \mathrm{CI}$ & $p$-Value ${ }^{\dagger}$ & $\mathbf{O R} *$ & $95 \% \mathrm{CI}$ & $p$-Value ${ }^{\dagger}$ \\
\hline Necessity of regular cancer screening & 1.29 & $(1.08-1.55)$ & 0.005 & 0.96 & $(0.78-1.19)$ & 0.695 \\
\hline Necessity of cancer screening from the age of 20 & 1.07 & $(0.89-1.30)$ & 0.464 & - & - & - \\
\hline Cervical cancer negatively affects pregnancy & 1.33 & $(1.11-1.60)$ & 0.002 & 1.08 & $(0.87-1.34)$ & 0.504 \\
\hline Cervical cancer may cause death & 1.14 & $(0.97-1.35)$ & 0.112 & - & - & - \\
\hline $\begin{array}{l}\text { The virus leading to cervical cancer } \\
\text { infects during sexual intercourse }\end{array}$ & 1.19 & $(1.00-1.42)$ & 0.048 & 0.872 & $(0.71-1.07)$ & 0.19 \\
\hline The virus leading to cervical cancer is HPV & 1.21 & $(0.99-1.47)$ & 0.064 & - & - & - \\
\hline $\begin{array}{l}\text { HPV vaccination is effective } \\
\text { for cervical cancer prevention }\end{array}$ & 2.03 & $(1.67-2.49)$ & $<0.001$ & 2.15 & $(1.69-2.74)$ & $<0.001$ \\
\hline
\end{tabular}

${ }^{*}$ Logistic regression analysis; ${ }^{+}$Chi-squared test.

\subsection{Sexual Activity and Behavior}

Analyses of the items related to sexual debut, sexual partners, and sexually transmitted infections were restricted to experienced women $(n=283)$. Analyses of condom use and pregnancy were restricted to unmarried and experienced women $(n=247)$ (Table 3). Age at sexual debut was significantly lower in the unvaccinated group compared to the 
vaccinated group, with $15.8( \pm 2.4)$ years versus $16.6( \pm 2.2)$ years, respectively $(p=0.008)$. The number of experienced individuals was also significantly higher in the unvaccinated group compared to the vaccinated group, with $7.0( \pm 12.4)$ versus $4.0( \pm 6.2)$, respectively $(p=0.016)$. Among all the 828 individuals, $232(28.0 \%)$ reported meeting men via the Internet, with significantly $(p=0.0032)$ more women in the unvaccinated group $(n=134$; $32.4 \%)$ than in the vaccinated group $(n=98 ; 23.7 \%)$ having done so. The significant difference was identified for condom use, though there were more women in the vaccinated group who said they used a condom "every time" or "frequently" $(83.7 \%, p=0.046)$. Pregnancy was significantly more prevalent in the unvaccinated group compared to the vaccinated group, with 14 people $(11.9 \%)$ versus $6(4.7 \%)$, respectively $(p=0.036)$. In terms of history of sexually transmitted infections, no significant differences were observed $(p=0.72)$.

Table 3. Sexual activity and behavior.

\begin{tabular}{|c|c|c|c|}
\hline & Vaccinated $(n=414)$ & Unvaccinated $(n=414)$ & $p$-Value \\
\hline Age at sexual debut ${ }^{1}($ mean \pm SD) & $16.6 \pm 2.2$ & $15.8 \pm 2.4$ & $0.008^{+}$ \\
\hline Number of previous sexual partners ${ }^{1}$ (mean \pm SD) & $4.0 \pm 6.2$ & $7.0 \pm 12.4$ & $0.016 \ddagger$ \\
\hline \multicolumn{4}{|l|}{ Experience of meeting a men via the Internet } \\
\hline Yes & $98(23.7 \%)$ & $134(32.4 \%)$ & $0.003 *$ \\
\hline No & $314(75.8 \%)$ & $269(65.0 \%)$ & \\
\hline Unanswered & $2(0.5 \%)$ & $11(2.7 \%)$ & \\
\hline \multicolumn{4}{|l|}{ Condom use ${ }^{2}$} \\
\hline Every time & $80(62.0 \%)$ & $56(47.5 \%)$ & $0.046 \S$ \\
\hline Frequent & $28(21.7 \%)$ & $30(25.4 \%)$ & \\
\hline Occasional & $9(7.0 \%)$ & $17(14.4 \%)$ & \\
\hline Seldom & $2(1.6 \%)$ & $9(7.6 \%)$ & \\
\hline Never & $8(6.2 \%)$ & $3(2.5 \%)$ & \\
\hline Unanswered & $2(1.6 \%)$ & $3(2.5 \%)$ & \\
\hline \multicolumn{4}{|l|}{ Experience of pregnancy ${ }^{2}$} \\
\hline Yes & $6(4.7 \%)$ & $14(11.9 \%)$ & $0.036^{*}$ \\
\hline No & $123(95.3 \%)$ & $99(83.9 \%)$ & \\
\hline Unanswered & $0(0.0 \%)$ & $5(3.4 \%)$ & \\
\hline \multicolumn{4}{|l|}{ Past history of sexually transmitted infections ${ }^{1}$} \\
\hline Yes & $10(7.4 \%)$ & $14(9.5 \%)$ & $0.72 *$ \\
\hline No & $124(91.9 \%)$ & $132(89.2 \%)$ & \\
\hline Unanswered & $1(0.7 \%)$ & $2(1.4 \%)$ & \\
\hline
\end{tabular}

${ }^{1}$ experienced women $(n=283) ;{ }^{2}$ unmarried and experienced women $(n=247) ;{ }^{*}$ Chi-square test (exclude unanswered); ${ }^{\dagger} t$-test; ${ }^{\ddagger}$ MannWhitney test.; $§$ Chi-squared test ("“"every time" and "frequent"“ vs. "“'occasional", "seldom" and "never" ", exclude unanswered).

\subsection{Change in Awareness of Sexual Activity after HPV Vaccination}

We asked about changes in awareness and actions regarding contraception, free sexual behavior, and sexually transmitted diseases after inoculation (Figure 1). Results showed that those who answered "I didn't pay too much attention to contraception" or "I came to have sex freely" after vaccination were extremely few $(n=2 ; 0.5 \%)$. There were $16(3.9 \%)$ women who answered "Anxiety about sexually transmitted diseases disappeared," 30 $(7.2 \%)$ who answered "I thought that I was old enough to have sex with men," and over $90 \%$ of women who did not observe any obvious changes in awareness of sexual activity following vaccination.

Over $90 \%$ of women did not observe any obvious changes in awareness of sexual activity following vaccination. Those who answered "I didn't pay too much attention to contraception" or "I came to have sex freely" after vaccination were extremely few $(n=2 ; 0.5 \%)$. 


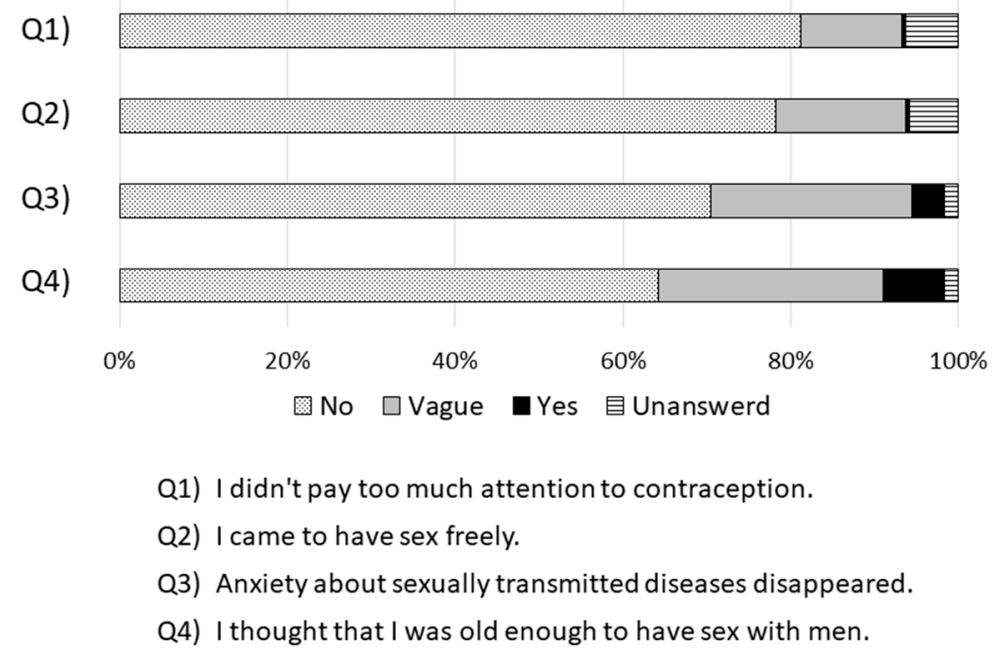

Figure 1. Change in awareness of sexual activity after HPV vaccination.

\subsection{Anxiety and Preventive Action against Cervical Cancer}

Results for anxiety about cervical cancer and sexually transmitted diseases and preventive action against cervical cancer are shown in Table 4 . No significant differences were observed between the vaccinated and unvaccinated groups for anxiety associated with cervical cancer $(p=0.48)$ or sexually transmitted diseases $(p=0.15)$. No significant differences were observed between the two groups regarding intent to regularly undergo cervical cancer screening $(p=0.62)$, but the percentage of those who agreed with the statement "Vaccination is preferable if it can prevent cancer" was significantly higher in the vaccinated group compared to the unvaccinated group $(p<0.001)$.

Table 4. Anxiety and preventive action against cervical cancer.

\begin{tabular}{|c|c|c|c|}
\hline Question & Vaccinated $(n=414)$ & Unvaccinated $(n=414)$ & $p$-Value \\
\hline \multicolumn{4}{|c|}{ Are you afraid you might get cervical cancer? } \\
\hline terribly & $139(33.6 \%)$ & $125(30.2 \%)$ & $0.48 *$ \\
\hline yes & $171(41.3 \%)$ & $175(42.3 \%)$ & \\
\hline no & $85(20.5 \%)$ & $86(20.8 \%)$ & \\
\hline unknown & $19(4.6 \%)$ & $28(6.8 \%)$ & \\
\hline \multicolumn{4}{|c|}{ Are you afraid you might get sexually transmitted diseases? } \\
\hline terribly & $136(32.9 \%)$ & $107(25.8 \%)$ & $0.15 *$ \\
\hline yes & $135(32.6 \%)$ & $143(34.5 \%)$ & \\
\hline no & $143(34.5 \%)$ & $164(39.6 \%)$ & \\
\hline \multicolumn{4}{|c|}{ Are you willing to have cancer screening regularly? } \\
\hline definitely & $17(4.1 \%)$ & $23(5.6 \%)$ & $0.62^{+}$ \\
\hline probably & $250(60.4 \%)$ & $234(56.5 \%)$ & \\
\hline no & $85(20.5 \%)$ & $91(22.0 \%)$ & \\
\hline unknown & $62(15.0 \%)$ & $66(15.9 \%)$ & \\
\hline \multicolumn{4}{|c|}{ Vaccination is better way if vaccination can prevent cancer } \\
\hline agree & $301(72.7 \%)$ & $223(53.9 \%)$ & $0.001 \ddagger$ \\
\hline dysagree & $33(8.0 \%)$ & $54(13.0 \%)$ & \\
\hline unknown & $80(19.3 \%)$ & $137(33.1 \%)$ & \\
\hline
\end{tabular}

* Chi-squared test ("terribly" and "yes"“" vs. "no"); ${ }^{\dagger}$-test (exclude unknown); ${ }^{\ddagger}$ Chi-squared test (exclude unknown).

\section{Discussion}

This survey focused not only on comparisons between the groups of women who had and had not received a prior HPV vaccination, but also on the respective changes in awareness and behavior towards cervical cancer prevention and sexual behavior. The survey revealed three points. The first one is that young Japanese women have poor knowledge 
about cervical cancer and HPV. The second is that those in the unvaccinated group have a particularly poor knowledge of the subject and tend to have higher sexual activity. The final one is that only a minority of women experienced changes in awareness about sex and developed sexual activity after vaccination. Such a large-scale survey has not been previously reported in Japan and is the first report in Japan analyzing the association between HPV vaccination and sexual activity in girls.

Some opponents of the recommendations for HPV vaccination argue that increased sexual activity of the girls who have received HPV vaccination and their failure to seek cervical cancer screening care may conversely increase the risk of cervical cancer [16]. There have been international reports that parents are concerned about the effects of HPV vaccination on sexual activity in children [17], while others have reported that HPV vaccination does not affect sexual activity [18-20]. In Japan, there have been no previous report which investigated how awareness about sex and sexual activity changed after $\mathrm{HPV}$ vaccination. The current study showed no apparent increase in "changes in sexual activity" among the girls who received HPV vaccination such as altered awareness of sex and increased risks of HPV infection. Only two $(0.5 \%)$ respondents said that they became sexually active and no longer worried about contraception after vaccination, and the majority responded that they did not change their sexual behavior after vaccination. The results did not show that those in the vaccinated group had riskier sexual behaviors compared to those in the unvaccinated group. Instead, it was the unvaccinated group that tended to have riskier sexual behavior. The unvaccinated group was recognized as a high-risk group for cervical cancer not only because they were not vaccinated but also because of their sexual behavior. Correlations with socioeconomic status have been reported as factors associated with higher sexual risks [21,22]. It is difficult to assess socioeconomic status using an Internet survey for teenage women; this survey also found it difficult to obtain information on family income (unknown: $66 \%$ ). We were able to clarify socioeconomic differences between the groups by using the attendance of cram schools as a surrogate measure. Several studies have shown that sexual activity and education were correlated [23-25]; these findings support our results.

As for awareness of cervical cancer screening, there were significant differences between the vaccinated and unvaccinated groups for anxiety towards cervical cancer and intention to regularly undergo cervical cancer screening; the results did not indicate any negative impact of HPV vaccination on the intent to receive cervical cancer screening. According to the correlations between HPV vaccination and intention to undergo cervical cancer screening reported in Japan, more HPV-vaccinated females tend to undergo cervical cancer screening than unvaccinated women [26]. As such, promoting HPV vaccination, which is a form of primary prevention, in combination with increasing rates of cervical cancer screening, which is a form of secondary prevention, is crucial in eliminating cervical cancer.

Sex education in Japan grew alongside the mounting anxiety about AIDS in the 1980s, and course guidelines were revised in 1992 to continue the education process from elementary school to high school [27]. In 2002, however, a Diet member noted the description of oral contraceptives written in the accessory teaching materials on sex education and criticized the "remarkable absence of perspectives on the sanctity of sexual intercourse in fostering life," resulting in a severe pushback on sex education across Japan and serious reductions in sex education content in course guidelines in 2004-2018. Afterwards, there were increased concerns that sex education would increase sexual awareness in children and decrease the age at which sexual behavior would start; learning guidelines with reduced content are still used today [28,29]. The United Nations Educational, Scientific, and Cultural Organization (UNESCO) collaborated with the World Health Organization to conduct a worldwide survey of sex education and concluded that comprehensive sex education would not hasten sexual behavior, but in fact, bring about more prudent sexual behavior. As a result, an International Sexuality Education Guidance was developed by 
UNESCO in 2009 to provide effective sex education, and this has established a global institutional basis for sex education [30].

In our survey, young women were asked about their knowledge of cervical cancer and $\mathrm{HPV}$, and their levels of familiarity were lower than expected. The vaccinated group tended to have more knowledge of cervical cancer and HPV, but "Cervical cancer negatively affects pregnancy" was the only statement which more than half of the participants reported being "familiar" with. This were thought to be caused by problems in sex education guidance in schools—as mentioned above - and inadequate information regarding cervical cancer and its causes provided by attending physicians during vaccinations. It is presumed that girls who are vaccinated do so without knowing what they are being vaccinated for despite HPV vaccination being individualized by health facilities. This may be because approximately $80 \%$ of the vaccinated respondents were inoculated in medical and pediatric departments that do not treat cervical cancer. In the United Kingdom, parents or individuals are required to be fully informed before vaccination; vaccinating healthcare professionals must know about cervical cancer even for school-based inoculations. Similarly, Japan must provide accurate medical information not only to patients, but also to medical workers and, in its quest to reinitiate proactive recommendations, create a system that sufficiently provides explanations during vaccination.

There are three primary limitations to this survey. The first is that vaccination history was self-reported and may have been misclassified. Matching the archived immunization records showed a low self-reported negative predictive value of approximately $50 \%$; of those who responded that they did not receive HPV vaccination, $11 \%$ of girls were vaccinated (i.e., they forgot about their three inoculations) [31]. In other words, there are limits to the accuracy of inoculation information in the questionnaire survey. Inoculation records in Japan are managed by approximately 1700 municipalities, and the lack of integration at the national level makes the inoculation records difficult to examine. This issue is a major challenge for vaccine administration in Japan for accurately grasping the adverse reactions that might occur after HPV vaccination and advancing discussions toward reinitiating proactive recommendations for HPV vaccination and NIP introduction of nine-valent vaccines. The second is that sexual activity could not be accurately assessed because of the considerable number of married individuals in the unvaccinated group. As a countermeasure, we believe that some biases could be eliminated by conducting analyses on contraception and pregnancy only among unmarried individuals. The third is that the survey targeted women aged 16-20 years who received vaccination at public expense at that time. Since this age is not target for cervical cancer screening, there may be a tendency for women of this age to be less interested in cervical cancer and cervical cancer screening.

Japan is a monoethnic nation, and Japanese people follow national policies and behave similarly to those around them [32]. It is important to disseminate and provide accurate data on the efficacy and adverse reactions of vaccines to parents, schools, and municipalities to re-disseminate HPV vaccination in Japan in the future. Society must also be informed that alterations in awareness and behavior-such as increased sexual activity and loss of cervical cancer screening_-rarely occur among vaccinated girls.

\section{Conclusions}

In conclusion, not only do unvaccinated individuals not benefit from vaccines, but they also tend to engage in high-risk sexual behavior, and thus it is even more important to provide information on the effectiveness of vaccines and the usefulness of cancer screening in these groups. Measures such as improving sex education in compulsory education, providing accurate information on cervical cancer and HPV vaccination for vaccinated girls by physicians in charge of vaccination, and creating materials and systems for them are needed to reinitiate positive recommendations for subsequent dissemination of HPV vaccination.

Supplementary Materials: The following are available online at https:/ / www.mdpi.com/2076-393 X/9/2/87/s1, Table S1: Knowledge of cervical cancer screening and HPV vaccination. 
Author Contributions: Conceptualization, M.S., Y.U., A.Y., S.A., E.M., and T.E.; data curation, R.K.; formal analysis, R.K.; funding acquisition, T.E.; methodology, R.K., Y.U., and A.Y.; project administration, M.S.; supervision, T.E.; validation, M.Y., M.H., S.J.B.H., and M.K.; writing-original draft, R.K. and M.S.; writing - review and editing, M.Y. All authors have read and agreed to the published version of the manuscript.

Funding: This work was supported by the Health and Labor Sciences Research Grant No. 26272001 and the Japanese Agency for Medical Research and Development (JP15ck0106103).

Institutional Review Board Statement: The study was conducted according to the guidelines of the Declaration of Helsinki, and approved by the Niigata University Graduate School of Medical and Dental Sciences (Approval Number 2015-1866, 5 March 2014).

Informed Consent Statement: Informed consent was obtained from all subjects involved in the study.

Data Availability Statement: The data presented in this study are available on request from the corresponding author. The data are not publicly available due to restriction of privacy.

Conflicts of Interest: M.Y. received lecture fees from Merck Sharp and Dohme. T.E. received lecture fees from Merck Sharp and Dohme. Y.U. received lecture fees from GlaxoSmithKline/Japan Vaccine and Merck Sharp and Dohme, as well as research funds from Merck Sharp and Dohme. E.M. received honoraria and lecture fees from Roche Diagnostics and Merck Sharp and Dohme, as well as grants from Merck Sharp and Dohme. M.H. received lecture fees from GlaxoSmithKline/JapanVaccine, Merck Sharp and Dohme, and Sanofi Pasteur Inc. All other authors report no potential conflicts. The funders had no role in the design of the study; in the collection, analyses, or interpretation of data; in the writing of the manuscript, or in the decision to publish the results.

\section{References}

1. Kudo, R.; Yamaguchi, M.; Sekine, M.; Adachi, S.; Ueda, Y.; Miyagi, E.; Hara, M.; Hanley, S.J.B.; Enomoto, T. Bivalent human papillomavirus vaccine effectiveness in a japanese population: High vaccine-type-specific effectiveness and evidence of crossprotection. J. Infect. Dis. 2019, 219, 382-390. [CrossRef] [PubMed]

2. Hanley, S.J.B.; Yoshioka, E.; Ito, Y.; Kishi, R. HPV vaccination crisis in Japan. Lancet 2015, 385, 2571. [CrossRef]

3. Nakagawa, S.; Ueda, Y.; Yagi, A.; Ikeda, S.; Hiramatsu, K.; Kimura, T. Corrected human papillomavirus vaccination rates for each birth fiscal year in Japan. Cancer Sci. 2020, 111, 2156-2162. [CrossRef] [PubMed]

4. Sekine, M.; Kudo, R.; Yamaguchi, M.; Hanley, S.J.B.; Hara, M.; Adachi, S.; Ueda, Y.; Miyagi, E.; Ikeda, S.; Yagi, A.; et al. Japan's ongoing crisis on HPV vaccination. Vaccines 2020, 8, 362. [CrossRef]

5. Yagi, A.; Ueda, Y.; Ikeda, S.; Sekine, M.; Nakayama, T.; Miyagi, E.; Enomoto, T. Evaluation of future cervical cancer risk in Japan, based on birth year. Vaccine 2019, 37, 2889-2891. [CrossRef]

6. Ikeda, S.; Ueda, Y.; Hara, M.; Yagi, A.; Kitamura, T.; Kitamura, Y.; Konishi, H.; Kakizoe, T.; Sekine, M.; Enomoto, T.; et al. Human papillomavirus vaccine to prevent cervical intraep-ithelial neoplasia in Japan: A nationwide case-control study. Cancer Sci. 2020. [CrossRef]

7. Palmer, T.; Wallace, L.; Pollock, K.G.; Cuschieri, K.; Robertson, C.; Kavanagh, K.; Cruickshank, M. Prevalence of cervical disease We confirmed itat age 20 after immunisation with bivalent HPV vaccine at age 12-13 in Scotland: Retrospective population study. BMJ 2019, 365, 1161. [CrossRef]

8. Benard, V.B.; Castle, P.E.; Jenison, S.A.; Hunt, W.C.; Kim, J.J.; Cuzick, J.; Lee, J.-H.; Du, R.; Robertson, M.; Norville, S.; et al. Population-based incidence rates of cervical intraepithelial neoplasia in the human papillomavirus vaccine era. JAMA Oncol. 2017, 3, 833-837. [CrossRef]

9. Lei, J.; Ploner, A.; Elfström, K.M.; Wang, J.; Roth, A.; Fang, F.; Sundström, K.; Dillner, J.; Sparén, P. HPV Vaccination and the risk of invasive cervical cancer. New Engl. J. Med. 2020, 383, 1340-1348. [CrossRef]

10. Arbyn, M.; Xu, L. Efficacy and safety of prophylactic HPV vaccines: A Cochrane review of randomized trials. Expert Rev. Vaccines 2018, 17, 1085-1091. [CrossRef]

11. WHO. HPV Vaccines and Safety. Available online: https://www.who.int/immunization/hpv/vaccines/en/ (accessed on 20 January 2021).

12. Ciavattini, A.; Giannella, B.; de Vincenzo, R.; di Giuseppe, J.; Papiccio, M.; Lukic, A.; Carpini, G.D.; Perino, A.; Frega, A.; Sopracordevole, F.; et al. HPV Vaccination: The position paper of the italian society of colposcopy and cervico-vaginal pathology (SICPCV). Vaccines 2020, 8, 354. [CrossRef] [PubMed]

13. Liverani, C.A.; di Giuseppe, J.; Giannella, L.; Delli Carpini, G.; Ciavattini, A. Cervical cancer screening guidelines in the postvac-cination era: Review of the literature. J. Oncol. 2020, 2020. [CrossRef] [PubMed]

14. Motoki, Y.; Mizushima, S.; Taguri, M.; Takahashi, K.; Asano, R.; Kato, H.; Asai-Sato, M.; Katayama, K.; Okamoto, N.; Hirahara, F.; et al. Increasing trends in cervical cancer mortality among young Japanese women below the age of 50 years: An analysis using the kanagawa population-based cancer registry, 1975-2012. Cancer Epidemiol. 2015, 39, 700-706. [CrossRef] [PubMed] 
15. Cancer Registry and Statistics. Cancer Information Service, National Cancer Center, Japan. Available online: https://www.ncc. go.jp/en/cis/divisions/stat/index.html (accessed on 2 September 2020).

16. Suryadevara, M.; Handel, A.; Bonville, C.A.; Cibula, D.A.; Domachowske, J.B. Pediatric provider vaccine hesitancy: An un-derrecognized obstacle to immunizing children. Vaccine 2015, 33, 6629-6634. [CrossRef] [PubMed]

17. Scarinci, I.C.; Garcés-Palacio, I.C.; Partridge, E.E. An examination of acceptability of HPV Vaccination among african american women and latina immigrants. J. Women's Health 2007, 16, 1224-1233. [CrossRef] [PubMed]

18. Brouwer, A.F.; Delinger, R.L.; Eisenberg, M.C.; Campredon, L.P.; Walline, H.M.; Carey, T.E.; Meza, R. HPV vaccination has not increased sexual activity or accelerated sexual debut in a college-aged cohort of men and women. BMC Public Health 2019, 19, 1-8. [CrossRef]

19. Chua, G.T.; Ho, F.K.; Tung, K.T.; Wong, R.S.; Cheong, K.N.; Yip, P.S.; Fan, S.Y.; Wong, W.H.; Qiao, Y.; Chui, C.S.; et al. Sexual behaviors and intention for cervical screening among HPV-vaccinated young Chinese females. Vaccine 2020, 38, 1025-1031. [CrossRef]

20. Mascaro, V.; Pileggi, C.; Currà, A.; Bianco, A.; Pavia, M. HPV vaccination coverage and willingness to be vaccinated among 18-30 year-old students in Italy. Vaccine 2019, 37, 3310-3316. [CrossRef]

21. Santelli, J.; Lowry, R.; Brener, N.D.; Robin, L. The association of sexual behaviors with socioeconomic status, family structure, and race/ethnicity among US adolescents. Am. J. Public Health 2000, 90, 1582-1588. [CrossRef]

22. Muñoz, D.-R.; Wellings, K.; Torres, E.C.; Dardet, C.A.; Cases, M.C.; Pérez, P. Sexual health and socioeconomic-related factors in Spain. Ann. Epidemiol. 2013, 23, 620-628. [CrossRef]

23. Drolet, M.; Boily, M.-C.; Greenaway, C.; Deeks, S.L.; Blanchette, C.; Laprise, J.-F.; Brisson, M. Sociodemographic Inequalities in sexual activity and cervical cancer screening: Implications for the success of human papillomavirus vaccination. Cancer Epidemiol. Biomark. Prev. 2013, 22, 641-652. [CrossRef] [PubMed]

24. Samandari, G.; Speizer, I.S. Adolescent sexual behavior and reproductive outcomes in Central America: Trends over the past two decades. Int. Perspect. Sex. Reprod. Health 2010, 36, 26-35. [CrossRef] [PubMed]

25. White, C.N.; Warner, L.A. Influence of family and school-level factors on age of sexual initiation. J. Adolesc. Health 2015, 56, $231-237$. [CrossRef] [PubMed]

26. Taniguchi, M.; Ueda, Y.; Yagi, A.; Ikeda, S.; Endo, M.; Tomimatsu, T.; Nakayama, T.; Sekine, M.; Enomoto, T.; Kimura, T. Cervical cancer screening rate differs by HPV vaccination status: An interim analysis. Vaccine 2019, 37, 4424-4426. [CrossRef]

27. Nishioka, E. Historical transition of sexuality education in Japan and outline of reproductive health/rights. Nippon. Eiseigaku Zasshi 2018, 73, 178-184. [CrossRef]

28. Ministry of Health, Labour and Welfare. Available online: http://sukoyaka21.jp/about (accessed on 2 September 2020).

29. Ministry of Education, Culture, Sports, Science and Technology. The Courses of Study for Upper Secondary Schools. Health \& Physical Education. Available online: https:/ / www.mext.go.jp/content/1407073_07_1_2.pdf (accessed on 2 September 2020).

30. The United Nations Educational, Scientific and Cultural Organization. International Technical Guidance on Sexuality Education: An Evidence-Informed Approach; The United Nations Educational, Scientific and Cultural Organization: Paris, France, 2018. Available online: https://unesdoc.unesco.org/ark:/48223/pf0000260770 (accessed on 2 September 2020).

31. Yamaguchi, M.; Sekine, M.; Kudo, R.; Adachi, S.; Ueda, Y.; Miyagi, E.; Hara, M.; Hanley, S.J.; Enomoto, T. Differential misclassification between self-reported status and official HPV vaccination records in Japan: Implications for evaluating vaccine safety and effectiveness. Papillomavirus Res. 2018, 6, 6-10. [CrossRef]

32. Triandis, H.C.; Suh, E.M. Cultural influences on personality. Annu. Rev. Psychol. 2002, 53, 133-160. [CrossRef] 